\section{Original Article}

Check for updates

\section{OPEN ACCESS}

Received: Jul 27, 2018

Revised: Nov 8, 2018

Accepted: Nov 18, 2018

Correspondence to

Dong In Suh, MD, PhD

Department of Pediatrics, Seoul National

University College of Medicine, 101 Daehak-ro,

Jongno-gu, Seoul 03080, Korea.

Tel: +82-2-2072-3625

Fax: +82-2-743-3455

E-mail: dongins0@snu.ac.kr

Copyright (c) 2019 The Korean Academy of Asthma, Allergy and Clinical Immunology . The Korean Academy of Pediatric Allergy and Respiratory Disease

This is an Open Access article distributed under the terms of the Creative Commons Attribution Non-Commercial License (https:// creativecommons.org/licenses/by-nc/4.0/) which permits unrestricted non-commercial use, distribution, and reproduction in any medium, provided the original work is properly cited.

ORCID iDs

Hea Lin Oh iD

https://orcid.org/0000-0002-6583-8993

Dong Yoon Kang (ID)

https://orcid.org/0000-0003-4283-2633

Hye-Ryun Kang (D)

https://orcid.org/0000-0002-2317-4201

sujeong Kim (iD

https://orcid.org/0000-0002-2494-9216

Young-Il Koh (iD

https://orcid.org/0000-0002-5100-9473

Sae Hoon Kim (iD)

https://orcid.org/0000-0002-2572-5302

Min-Hye Kim (iD)

https://orcid.org/0000-0002-1775-3733

Dong In Suh (D)

https://orcid.org/0000-0002-7817-8728

\title{
Severe Cutaneous Adverse Reactions in Korean Pediatric Patients: A Study From the Korea SCAR Registry
}

\author{
Hea Lin Oh $\left(\mathbb{D},{ }^{1}\right.$ Dong Yoon Kang $\left(\mathbb{D},{ }^{2,3}\right.$ Hye-Ryun Kang $\left(\mathbb{1},{ }^{4,5}\right.$ Sujeong Kim $\left(\mathbb{D},{ }^{6}\right.$ \\ Young-Il Koh (1),7 Sae Hoon Kim $\left(\mathbb{D},{ }^{8}\right.$ Min-Hye Kim (1), ${ }^{9}$ Dong In Suh $\left(\mathbb{1},,^{*}\right.$ and \\ the Korean Severe Cutaneous Adverse Reactions Consortium
}

\begin{abstract}
'Department of Pediatrics, Seoul National University College of Medicine, Seoul, Korea ${ }^{2}$ Drug Safety Monitoring Center, Seoul National University Hospital, Seoul, Korea

${ }^{3}$ Department of Preventive Medicine, Seoul National University College of Medicine, Seoul, Korea ${ }^{4}$ Department of Internal Medicine, Seoul National University Hospital, Seoul, Korea

${ }^{5}$ Institute of Allergy and Clinical Immunology, Seoul National University Medical Research Center, Seoul, Korea ${ }^{6}$ Department of Internal Medicine, School of Medicine, Kyungpook National University, Daegu, Korea ${ }^{7}$ Department of Internal Medicine, Chonnam National University Medical School, Gwangju, Korea ${ }^{8}$ Department of Internal Medicine, Seoul National University Bundang Hospital, Seongnam, Korea ${ }^{9}$ Department of Internal Medicine, College of Medicine, Ewha Womans University, Seoul, Korea
\end{abstract}

\section{ABSTRACT}

Purpose: Although severe cutaneous adverse drug reactions (SCARs) are rare, they are associated with high morbidity and mortality, and thus early diagnosis and treatment are critical for improving prognoses. However, few studies have reported the characteristics of SCARs in children. Thus, we aimed to evaluate the clinical characteristics, current management and prognosis of pediatric SCARs.

Methods: We analyzed pediatric data in the Korean SCARs registry, which was built retrospectively in 2016 with SCAR cases treated in 34 tertiary referral university hospitals during 2010-2015. Using these cases, we descriptively analyzed detailed data regarding etiology, clinical and laboratory features, treatment strategies, and prognosis.

Results: Forty-seven pediatric SCAR cases from 15 tertiary referral hospitals were included. The median patient age was 10 (interquartile range, $3-15.5)$ years and $68.1 \%(n=32)$ were males. The culprit drug was identified in $95.7 \%(n=45)$ of the patients; antibiotics $(44.7 \%)$ and antiepileptic drugs (19.1\%) were the most common and second most common culprits, respectively. Drug Reaction with Eosinophilia and Systemic Symptoms (DRESS) cases presented with the largest area of skin involvement without permanent sequelae. StevensJohnson syndrome (SJS) cases involved relatively small areas of skin but serious sequelae in two children. Of 4 patients with toxic epidermal necrolysis (TEN), 1 died. Of all patients assessed, $36(76.6 \%)$ received systemic steroids and $21(44.7 \%)$ received intravenous immunoglobulin (IVIG). Thirteen (27.7\%) received both systemic steroids and IVIG. Cyclosporine was administered to only 1 patient along with a systemic steroid. Conclusions: In patients with pediatric SCARs, including those with DRESS, SJS and TEN, clinical presentations were variable. Thus, there was no clear continuous disease spectrum. Although the mortality rate was low $(2.1 \%)$, clinical suspicion may be the best tool for proactive SCAR management.

Keywords: Drug-related side effects and adverse reactions; children; Drug eruptions 
Disclosure

There are no financial or other issues that might lead to conflict of interest.

\section{INTRODUCTION}

Cutaneous reactions constitute the most common type of adverse drug reactions in pediatric populations, with a prevalence of up to $36 \% .{ }^{1}$ Despite the high prevalence of cutaneous adverse drug reactions, these reactions are mostly benign, cause only mild clinical symptoms, and subside with discontinuation of the suspected drug. ${ }^{2}$ However, $2.0 \%-6.7 \%$ of cutaneous reactions can develop into severe cutaneous adverse drug reactions (SCARs), which are potentially life-threatening. ${ }^{3,4}$

Considering serious morbidity and mortality associated with SCARs, their early diagnosis and treatment is crucial for achieving better prognoses. As clinical suspicion of SCARs is often based on a patient's clinical history, it is essential to determine the frequency and common symptoms of SCARs.

Many previous studies have examined the clinical presentation and risk factors of SCARs in adults. ${ }^{5,6}$ However, few have reported these characteristics in a pediatric population, and even less in Korean children. This study aimed to retrospectively analyze the clinical characteristics, laboratory findings and clinical outcomes of pediatric SCAR cases using data from a large Korean clinical registry.

\section{MATERIALS AND METHODS}

Pediatric data was collected from the Korean SCARs registry, which was built in 2016 using retrospective data from 34 tertiary referral hospitals in 2010-2015. To build the registry, researchers uploaded their institutions' potential cases and 2 allergy specialists validated each SCAR case. Researchers from 34 hospitals first filtered the individual case safety reports on adverse drug reactions, medical records on the diagnosis, and consultation records with allergists and dermatologists using the keywords of 'Drug Reaction with Eosinophilia and Systemic Symptoms (DRESS),' 'Stevens-Johnson syndrome (SJS),' 'toxic epidermal necrolysis (TEN),' and 'drug hypersensitivity.' The medical records of the resulting filtered cases were then reviewed. All cases that meet the European Registry of SCARs (RegiSCAR) inclusion criteria were selected as the potential SCAR cases. ${ }^{7,8}$

Potential cases of SJS/TEN were determined as hospitalized patients with widespread exanthema with $1 \%$ or more of skin detachment of epidermis, or with more than 1 blister and mucous membrane involvement. Potential cases of DRESS were defined as hospitalized patients with acute onset of exanthema with fever $>38^{\circ} \mathrm{C}$, enlarged lymph nodes, involvement of at least 1 internal organ, and 1 or more of the following: eosinophilia $>10 \%,>700 / \mu \mathrm{L}$; atypical lymphocytes; lymphopenia < 4,000; lymphocytosis; or thrombopenia. Potential cases were excluded when symptoms and signs were suspicious of the following: pemphigus, erythema multiforme, bullous pemphigoid, staphylococcal scalded skin syndrome, mechanobullous eruption (heat, cold, friction, pressure), acute pustular psoriasis, Kawasaki's disease, toxic shock syndrome, graft-versus-host disease, vasculitis or epidermolysis bullosa.

The clinical data of potential cases were uploaded to the main data center with a standardized form that included data on the demographics, past medical history, culprit agents, clinical and laboratory findings, treatment, and clinical outcomes associated with each case. In addition, comorbid conditions were also investigated, including allergic diseases (asthma, 
rhinitis and atopic dermatitis), diabetes mellitus, renal and urologic diseases (acute cystitis, chronic renal failure, dialysis and kidney transplantation), hepatic diseases (hepatitis and cirrhosis), infectious diseases (human immunodeficiency virus [HIV] and other viral infection; pneumonia, bronchiolitis, rhinopharyngitis, otitis media, and tonsillitis), digestive diseases (gastroenteritis, acute cystitis), rheumatic diseases, immune deficiency, and malignancy.

Causality assessment of SCARs to culprit drugs was conducted according to the World Health Organization-Uppsala Monitoring Center (WHO-UMC) criteria. ${ }^{9}$ The 'probable' relationship was suspected when a SCAR event presented a reasonable time relationship to drug intake, was unlikely attributed to disease or other drugs, and responded well to withdrawal. The 'certain' causality was suspected when there was relevant rechallenge information on the case with 'probable' causality.

Uploaded data of all potential cases were also reviewed by another allergy specialist in the main center, and the final SCAR cases were confirmed by both specialists of the regional hospital and the main center. Cases were registered only when both specialists agreed upon the SCAR diagnosis and the causality of 'probable' or 'certain.' Then, to evaluate the clinical characteristics of pediatric SCAR cases, we selected cases of individuals who were aged $<19$ years.

A final diagnoses of SJS was made when clinical records indicated the following: acute onset of mucous membrane involvement of at least 2 mucosal surfaces; skin symptoms, including maculae, target-like, bullae or erosion; a positive Nikolsky sign; and epidermal detachment of less than $10 \%$ of the total body surface area. ${ }^{10} \mathrm{~A}$ final diagnosis of TEN was made by the presence of the same lesions as in SJS, but with a confluence of blisters leading to a positive Nikolsky sign and the detachment of large epidermal sheets on more than $30 \%$ of the body surface area.$^{10}$ Marginal cases between SJS and TEN were classified into SJS. A final diagnosis of DRESS was made based on the following items: characteristics of fever, enlarged lymph nodes, eosinophilia, atypical lymphocytes, skin involvement, organ involvement, duration for resolution and the exclusion of other potential causes. Since the scoring system for classifying DRESS of RegiSCAR ${ }^{8,11}$ and the diagnostic criteria of the Japanese consensus group ${ }^{12,13}$ are similar but different, at least 2 allergy specialists discussed each potential case and moderated the DRESS diagnosis on a case-by-case basis.

Statistical analyses were performed using SPSS 22.0 software for Windows (SPSS, Inc, Chicago, IL, USA). Descriptive statistics are provided and include the number and percentage for categorical variables and the median and interquartile range (IQR) for continuous variables. The protocol was approved by the Institutional Review Board of Seoul National University Hospital (No. 1804-115-939) and due to the retrospective nature of the study, the requirement for written informed consent was waived.

\section{RESULTS}

A total of 814 potential cases was listed. Thirty-three and 36 cases were excluded because of the onset time was out of 2010-2015 and doubtful diagnosis/causality, respectively. Among the 745 confirmed SCAR cases, a total of 47 occurred in subjects $<19$ years (Figure).

The patient characteristics of the total 47 SCAR cases are shown in Table 1. The median patient age was $10(\mathrm{IQR}, 3-15.5)$ years and $68.1 \%(\mathrm{n}=32)$ were male. Of all cases, $14.9 \%(\mathrm{n}=7)$ 
Cases reported to each regional pharmacovigilance center

Primary screening and listing based on the medical chart review

Prepare for analyzing the pediatirc cases

Secondary review of listed cases based on the uploaded data

Prepare for analyzing the pediatirc
cases




\section{Etiology}

The culprit drugs were identified in $95.7 \%(n=45)$ of the SCAR cases. The distribution of drug type, number and administration route across SCAR groups are listed in Table 2. Antibiotics were the most common (44.7\%) culprit drugs, while antiepileptic drugs were the second most common $(19.1 \%)$. Causative drugs for the remaining $4.3 \%(n=2)$ of the patients could not be identified.

In patients with DRESS, antibiotics (42.9\%) and antiepileptic drugs (57.1\%) played a major role in disease etiology. The most common causative antibiotic was amoxicillin/clavulanate (38.1\%); the second most common causative antibiotic was cephalosporin (28.6\%). In patients with SJS, antibiotics (41.7\%), antiepileptic drugs (18.9\%) and antipyretics (25.0\%) were found to be the 3 most common causative classes of drugs. Antibiotics (75\%) and systemic steroids (25\%) were suspected to cause all 4 cases of TEN.

Twenty-seven (57.4\%) patients were administered a single drug, while the other 20 (42.6\%) received 1 suspected drug together with another medication unrelated with SCARs. Fortythree patients ingested the drug(s) orally, 1 was administered the medication intravenously, and there was no information on administration route available for the remaining 3 patients.

\section{Clinical and laboratory features}

Case clinical and laboratory features are presented in Table 3 . The most common type of skin disorder was rash $(91.5 \%, n=43)$. In patients with SJS or TEN, other skin symptoms such

Table 2. Kind, number and the route of suspected drugs as the etiology

\begin{tabular}{|c|c|c|c|c|}
\hline Etiology & DRESS $(n=7)$ & SJS $(n=36)$ & $\operatorname{TEN}(n=4)$ & Total $(n=47)$ \\
\hline Antibiotics & $3(42.9)$ & $15(41.7)$ & $3(75.0)$ & $21(44.7)$ \\
\hline Penicillin & & 9 & 2 & 11 \\
\hline Amoxicillin/clavulate & & 7 & 1 & 8 \\
\hline TMP/SMX & & 2 & 1 & 3 \\
\hline Cephalosporine & 2 & 3 & 1 & 6 \\
\hline Macrolide & & 2 & & 2 \\
\hline Dapsone & 1 & & & 1 \\
\hline Doxycycline & & 1 & & 1 \\
\hline Antiepileptics & $4(57.1)$ & $5(13.9)$ & & 9 (19.1) \\
\hline Phenobarbital & 1 & 1 & & 2 \\
\hline Carbamazepine & 1 & 2 & & 3 \\
\hline Lamotrigine & 2 & 2 & & 4 \\
\hline NSAIDS & & $3(8.3)$ & & $3(6.4)$ \\
\hline Acetaminophen & & $6(16.7)$ & & $6(12.8)$ \\
\hline Other drugs & & $5(13.9)$ & $1(25.0)$ & $6(12.8)$ \\
\hline Deflazacort & & 1 & 1 & 2 \\
\hline Potassium clavulanate & & 1 & & 1 \\
\hline Guaifenesin & & 1 & & 1 \\
\hline Streptokinase & & 1 & & 1 \\
\hline Dacarbazine & & 1 & & 1 \\
\hline Unknown & & 2 & & $2(4.3)$ \\
\hline \multicolumn{5}{|l|}{ Medication numbers } \\
\hline 1 & $4(57.1)$ & $21(58.3)$ & $2(50.0)$ & $27(57.4)$ \\
\hline$\geq 2$ & $3(42.9)$ & $15(41.7)$ & $2(50.0)$ & $20(42.6)$ \\
\hline \multicolumn{5}{|l|}{ Administration route } \\
\hline Oral & $5(71.4)$ & $34(94.4)$ & $4(100)$ & $43(91.5)$ \\
\hline IV & $1(14.3)$ & & & $1(2.1)$ \\
\hline Unknown route & $1(14.3)$ & $2(5.6)$ & & $3(6.4)$ \\
\hline
\end{tabular}

Values are presented as number (\%).

DRESS, drug reaction with eosinophilia and systemic symptoms; SJS, Stevens-Johnson syndrome; TEN, toxic epidermal necrolysis; TMP/SMX, trimethoprim/ sulfamethoxazole; NSAIDS, nonsteroidal anti-inflammatory drugs; IV, intravenous. 
as appearance of the target sign $(14.9 \%, \mathrm{n}=17)$, desquamation $(21.3 \%, \mathrm{n}=10)$ and blister $(48.9 \%, n=23)$ were also observed. The Nikolsky sign was not observed in patients with DRESS but was observed in 5 patients (13.9\%) with SJS and in all 4 patients with TEN. In the DRESS group, the median extent of skin involvement was 99\% (IQR, 91\%-100\%), whereas in the SJS and TEN groups the median extent of skin involvement was 67.5\% (IQR, 37.1\%-100\%) and $99.5 \%$ (IQR, $97.9 \%-100 \%$ ), respectively.

In the DRESS group, 1 patient (14.3\%) experienced skin detachment. The detached skin area was $90 \%$ of the whole body on the first day of SCAR development and was fully recovered by the seventh day of SCAR development. In the SJS group, the median detached skin area was 3\% (IQR, 1.5\%-6.3\%) on the first day of SCAR development; the median decreased to $2 \%$ (IQR, $0 \%-5 \%$ ) on the seventh day. Six patients (16.7\%) had skin detachment on the first day of SCAR development. In the SJS group, 3 were fully recovered and 2 developed skin detachment on the seventh day of SCAR development. In the TEN group, 1 patient (25.0\%) had skin detachment. The detached skin area was $2 \%$ on the first day of SCAR development and progressed to $36 \%$ by the seventh day of SCAR development.

Table 3. Clinical and laboratory features of pediatric SCARs

\begin{tabular}{|c|c|c|c|}
\hline Characteristic & DRESS $(n=7)$ & SJS $(n=36)$ & $\operatorname{TEN}(n=4)$ \\
\hline Cutaneous lesion extent (\%) & $99(91.0-100)$ & $67.5(37.1-100)$ & $99.5(97.9-100)$ \\
\hline \multicolumn{4}{|l|}{ Cutaneous findings } \\
\hline Rash & $5(71.4)$ & $34(94.4)$ & $4(100)$ \\
\hline Target sign & & 5 (13.9) & $2(50)$ \\
\hline Desquamation & & $8(22.2)$ & $2(50)$ \\
\hline Blister & & $20(55.6)$ & $3(75)$ \\
\hline Nikolsky signs & & $5(13.9)$ & $4(100)$ \\
\hline \multicolumn{4}{|l|}{ Skin detachment } \\
\hline Day 1 & $1(14.3)$ & $6(16.7)$ & $1(25.0)$ \\
\hline Day 7 & & $5(13.9)$ & $1(25.0)$ \\
\hline \multicolumn{4}{|l|}{ Tachycardia* } \\
\hline Day 1 & & $6(16.7)$ & $1(25.0)$ \\
\hline Day 7 & & & $2(50.0)$ \\
\hline Mucosa involvement & $1(14.3)$ & $33(91.7)$ & $4(100)$ \\
\hline Oral & 1 & 23 & 3 \\
\hline Labial & & 19 & 4 \\
\hline Ocular & & 12 & 4 \\
\hline Genital & & 6 & 2 \\
\hline Respiratory & & 4 & 1 \\
\hline Lymphadenitis & $1(14.3)$ & $1(2.8)$ & $2(50)$ \\
\hline Fever & $6(85.7)$ & $24(66.7)$ & $3(75)$ \\
\hline Fever duration (day) & $5.0(4.0-8.0)$ & $4.0(2.0-6.0)$ & $13(10-21.5)$ \\
\hline \multicolumn{4}{|l|}{ Hematologic finding } \\
\hline Leukocytosis $^{\dagger}$ & $3(42.9)$ & $2(5.6)$ & $1(25)$ \\
\hline Leukopenia $^{\ddagger}$ & $2(28.6)$ & $10(27.8)$ & $2(50)$ \\
\hline Atypical lymphocytosis§ & $2(28.6)$ & $2(5.6)$ & $1(25)$ \\
\hline Eosinophiliall & $3(42.9)$ & $4(11.1)$ & $3(75)$ \\
\hline Thrombocytopeniaף & $2(28.6)$ & $7(19.4)$ & $2(50)$ \\
\hline Liver function abnormality ${ }^{\star *}$ & $7(100)$ & $13(36.1)$ & $2(50)$ \\
\hline Renal function abnormality ${ }^{\dagger \dagger}$ & & $1(2.8)$ & $1(25)$ \\
\hline
\end{tabular}

Values are presented as median (IQR) or number (\%).

SCARs, severe cutaneous adverse reactions; DRESS, drug reaction with eosinophilia and systemic symptoms; SJS, Stevens-Johnson syndrome; TEN, toxic epidermal necrolysis; IQR, interquartile range.

${ }^{*}$ Tachycardia: heart rate $>160$ beats/min in 0-2 years and heart rate $>120$ beats/min in $>2$ years; ${ }^{\dagger}$ Leukocytosis: white blood cell count $>20,000 / \mu \mathrm{L}$; ${ }^{\ddagger}$ Leukopenia: white blood cell count < 4,000/ $\mu \mathrm{L} ;{ }^{\S}$ Atypical lymphocytosis: atypical lymphocyte $\geq 5 \%$; "Eosinophilia: eosinophil > 4\%; "Thrombocytopenia: platelet < 150,000/ $\mu \mathrm{L}$;

${ }^{* *}$ Abnormalities in liver function tests: serum level of alanine transaminase $>40 \mathrm{IU} / \mathrm{L}$; ${ }^{\dagger \dagger} \mathrm{Abnormalities}$ in renal function tests: glomerular filtration rate $<100 \mathrm{~mL} / \mathrm{min} / 1.73 \mathrm{~m}^{2}$. 
In addition, the mucosal epithelium was involved in $1(14.3 \%)$ case of DRESS, $33(91.7 \%)$ cases of SJS and all 4 cases of TEN. The most commonly involved mucosal site was the mouth $(57.4 \%, n=27)$, followed by the labia $(48.9 \%, n=23)$. Lymphadenitis was observed in 1 patient (14.3\%) with DRESS, 1 patient (2.8\%) with SJS, and in 2 patients (50\%) with TEN. Finally, 6 patients $(85.7 \%)$ with DRESS, 24 patients $(66.7 \%)$ with SJS, and $3(75 \%)$ patients with TEN experienced a fever. The median fever duration was 5 days (IQR, 4-8) in the DRESS group, 4 days (IQR, 2-6) in the SJS group, and 13 days (IQR, 10-21.5) in the TEN group.

Laboratory abnormalities were found in 4 (57.1\%) patients in the DRESS group, 17 (47.2\%) patients in the SJS group, and in all patients in the TEN group. The most common laboratory abnormality was eosinophilia and leukocytosis in the DRESS group $(42.9 \%, \mathrm{n}=3)$, leukopenia in the SJS group $(27.8 \%, \mathrm{n}=10)$ and eosinophilia in the TEN group $(75 \%, \mathrm{n}=3)$. On the other hand, liver enzyme abnormalities were noted in all patients in the DRESS group, $13(36.1 \%)$ patients in the SJS group, and 2 (50\%) patients in the TEN group. Renal function abnormalities were only noted in the SJS group and the TEN group (1 patient for each).

\section{Treatment}

The medications used to treat pediatric SCARs are listed in Table 4. Of all the patients examined, $36(76.6 \%)$ received systemic steroids and $21(44.7 \%)$ received intravenous immunoglobulin (IVIG). Thirteen patients $(27.7 \%)$ received both systemic steroids and IVIG. When patients were divided by treatment option according to type of SCAR, all patients with DRESS received systemic steroids and $3(42.9 \%)$ received additional IVIG. In the SJS group, 25 (69.5\%) patients received systemic steroids, 14 (38.9\%) patients received IVIG, and 6 $(16.7 \%)$ patients received both systemic steroids and IVIG. Only 1 patient $(2.1 \%)$ in the SJS group received cyclosporine concomitantly with systemic steroids. All 4 patients with TEN received both systemic steroids and IVIG. The most common steroids used to treat SCARs were methylprednisolone $(36.2 \%, \mathrm{n}=17)$ and prednisolone $(25.5 \%, \mathrm{n}=12)$. Dexamethasone $(10.6 \%, n=5)$, hydrocortisone $(2.1 \%, n=1)$ and deflazacort $(2.1 \%, n=1)$ were also used. The median duration of steroid treatment was 31 days (IQR, 13-134) in the DRESS group, 2.5 days (IQR, 0.0-9.0) in the SJS group, and 6 days (IQR, 5-22) in the TEN group.

Table 4. Medications to treat pediatric SCARS

\begin{tabular}{|c|c|c|c|c|}
\hline Characteristic & DRESS $(n=7)$ & SJS $(n=36)$ & $\operatorname{TEN}(n=4)$ & Total $(n=47)$ \\
\hline Steroid use only & $4(57.1)$ & $19(52.8)$ & & $23(48.9)$ \\
\hline Methylprednisolone & 3 & 10 & 4 & 17 \\
\hline Prednisolone & 3 & 9 & & 12 \\
\hline Dexamethasone & 1 & 4 & & 5 \\
\hline Hydrocortisone & & 1 & & 1 \\
\hline Deflazacort & 1 & 4 & & 5 \\
\hline IVIG use only & & $8(22.2)$ & & $8(17.0)$ \\
\hline IVIG + Steroid & $3(42.9)$ & $6(16.7)$ & $4(100)$ & $13(27.7)$ \\
\hline Cyclosporine & & $1(2.8)$ & & $1(2.1)$ \\
\hline Total steroid use days & $31(13-134)$ & $2.5(0.0-9.0)$ & $6(5-22)$ & \\
\hline Other medication & $2(28.6)$ & $14(38.9)$ & $1(25.0)$ & $17(36.2)$ \\
\hline Antibiotics & 1 & 3 & & 4 \\
\hline Acyclovir & & 1 & & 1 \\
\hline Mupirocin & & 1 & & 1 \\
\hline Antihistamine & & 8 & & 8 \\
\hline L-cartinine & 1 & & & 1 \\
\hline UDCA & & 1 & & 1 \\
\hline Fentanyl & & & 1 & 1 \\
\hline
\end{tabular}

Values are presented as number (\%) or median (IQR).

DRESS, drug reaction with eosinophilia and systemic symptoms; IQR, interquartile range; IVIG, Intravenous immunoglobulin; SCARs, severe cutaneous adverse reactions; SJS, Stevens-Johnson syndrome; TEN, toxic epidermal necrolysis; UDCA, ursodeoxycholic acid. 
Table 5. Progression and prognosis of the pediatric SCARs

\begin{tabular}{|c|c|c|c|c|}
\hline Characteristic & DRESS $(n=7)$ & SJS $(n=36)$ & $\operatorname{TEN}(n=4)$ & Total $(n=47)$ \\
\hline Latency time (day) & $23.5(17.3-32.8)$ & $4(1-13)$ & $6.5(0.8-16)$ & 7.5 (1.75-19.0) \\
\hline Duration of symptom (day) & $15(9.5-19)$ & $12(8-15)$ & $32.5(26.8-62)$ & $13(9.0-17.0)$ \\
\hline Duration of admission(day) & $12(9.5-16.5)$ & $10(6.8-12.3)$ & $30.5(24.3-60.5)$ & $10(7.0-15.0)$ \\
\hline ICU care & $1(14.3)$ & $1(2.8)$ & $2(50.0)$ & $4(8.5)$ \\
\hline \multicolumn{5}{|l|}{ Clinical outcome } \\
\hline Recovery & $7(100)$ & $33(91.7)$ & $2(50.0)$ & $42(89.4)$ \\
\hline Sequelae & & $2(5.6)$ & $1(25.0)$ & $3(6.4)$ \\
\hline Skin & & 2 & & 2 \\
\hline Skin/skin appendage & & & 1 & 1 \\
\hline Death & & & $1(25.0)$ & $1(2.1)$ \\
\hline
\end{tabular}

Values are presented as number (\%) or median (IQR).

SCARs, severe cutaneous adverse reactions; DRESS, drug reaction with eosinophilia and systemic symptoms; SJS, Stevens-Johnson syndrome; TEN, toxic epidermal necrolysis; ICU, intensive care unit; IQR, interquartile range.

Seventeen patients (36.1\%) required further treatment in addition to the treatments indicated above. One DRESS patient and 3 SJS patients required antibiotics for a concurrent infection.

One patient with DRESS required L-carnitine, 8 patients with SJS required antihistamines, and 1 required acyclovir. The other patient with TEN required fentanyl for pain control.

\section{Progression and prognosis}

The progression and prognosis of pediatric SCAR cases are shown in Table 5. The median latency between drug administration and symptom expression was 23.5 (IQR, 17.3-32.8) days in the DRESS group, 4.0 days (IQR, 1.0-13.0) in the SJS group and 6.5 days (IQR, 0.8-16.0) in the TEN group. The median duration of symptoms was 15.0 days (IQR, 9.5-19.0) in the DRESS group, 12.0 days (IQR, 8.0-15.0) in the SJS group and 32.5 days (IQR, 26.8-62.0) in the TEN group. The median duration of admission was 12.0 days (IQR, 9.5-16.5) in the DRESS group, 10.0 days (IQR, 6.8-12.3) in the SJS group and 30.5 days (IQR, 24.3-60.5) in the TEN group. One patient in the DRESS group, 1 patient in the SJS group, and 2 patients in the TEN group were transferred to the intensive care unit (ICU). In the DRESS and SJS groups, the patients spent 7 and 3 days in the ICU, respectively. The 2 patients in the TEN group spent 5.0 days and 14.0 days in the ICU. Three (6.4\%) patients experienced permanent skin and/or skin appendage sequelae. One of the 4 patients with TEN died.

\section{DISCUSSION}

This study found that SJS comprised the majority of the total 47 pediatric SCAR cases, followed by DRESS and TEN. The majority of patients were male, antibiotics and antiepileptics were the most common culprit drugs, and respiratory infections were the most common comorbidities. The clinical presentations of DRESS, SJS and TEN varied remarkably, and thus their clinical characteristics could not be explained along a single continuous disease spectrum. In this study, systemic steroids were the most common treatment of choice, and the mortality rate from pediatric SCARs was $2.1 \%$. This is the first study to describe the clinical characteristics, current treatment modalities and prognosis of pediatric SCAR cases in Korea.

The culprit drug for SCAR events in this study was identified in $95.7 \%$ of the total assessed cases, which is comparable to $93.1 \%$ in a large-scaled pediatric research. ${ }^{10}$ However, it is higher than that of adult cases where $30 \%$ of SJS/TEN cases presented no causative drug. ${ }^{14}$ Considering that SCAR can be induced by infections such as HIV, hepatitis virus, herpes virus 
and Mycoplasma pneumoniae, ${ }^{15}$ it can be possible that part of SCARs associated with infection may have been regarded as SCARs due to antibiotics or antipyretics in children.

In the present study, we found that commonly used drugs were often the cause of pediatric SCARs; this tendency was observed across all SCAR groups. Importantly, antibiotics and antipyretics are among the most commonly used drugs in children, ${ }^{16}$ and we found that beta-lactam and macrolide antibiotics were the most common SCAR culprits in the present study. Antiepileptics, which were also found to be associated with high rates of SCARs in this study, are not commonly used in children; however, when they have been prescribed, they are used chronically. Our findings are consistent with those of a previous study, which reported a tendency for children to be most frequently affected by commonly used drugs, while adults are affected most frequently by specific drugs such as allopurinol, neuroleptics or antihypertensives. ${ }^{17}$

One interesting finding of the present study was that $57.4 \%$ of the patients experienced pediatric SCARs from a single drug. This differs from that of a previous study which found that $92.6 \%$ of pediatric SCAR cases resulted from a single drug. ${ }^{6}$ Moreover, most cases of SCARs in the present study resulted from orally administered medications; only 1 was associated with intravenous drug administration. This finding also contrasts with that of a previous report, which found that SCARs tended to occur more frequently after non-oral drug administration..$^{18}$ Furthermore, 2 patients $(4.3 \%)$ developed SCARs while they took systemic steroid and, in 1 out of 4 cases of TEN, oral steroid was suspected, which was not expected in another large-scaled pediatric research. ${ }^{10}$ In the literature, the rate of steroid hypersensitivity reaction is as low as $0.1 \% 0.3 \%,{ }^{19,20}$ but the incidence of SCAR due to systemic steroid was not known. In a case-control study conducted by the EuroSCAR group, exposure to systemic steroid around the time of manifestation was as high as $14.8 \%$ in the SJS/TEN group..$^{21}$ In that study, however, $55 \%$ of subjects with steroid-associated SJS/TEN were already taking another concomitant medication that are classified as high-risk for SCARs. Therefore, it is true that the systemic steroid can cause SCARs, but the result needs to be cautiously interpreted.

It has been proposed that DRESS, SJS and TEN are not individual diseases but rather exist along a SCAR spectrum. ${ }^{22}$ However, our results showed that DRESS was associated with different clinical characteristics than SJS or TEN. In DRESS cases, the area of skin damage could be as high as $90 \%$, but resolved spontaneously and completely in 7 days. However, in SJS and TEN cases the area of skin damage was much smaller, but the damage did not recover in 7 days and left unresolved skin or mucosal damage. Furthermore, the involvement of the mucosa, most commonly in oral, labial and ocular zones, was shown in the SJS and TEN groups, but not in the DRESS group as has previously been reported. ${ }^{10}$

In the present study, we found that SCARs were accompanied by various systemic symptoms. This has previously been reported, ${ }^{17}$ but is some discrepancy between our results and previous findings. For example, while lymphadenitis was previously reported to be a common symptom of DRESS, ${ }^{10}$ it more frequently occurred with SJS and TEN in the SCAR cases that we analyzed. The pathogenesis of SCARs is not fully understood. However, considering that SCAR is associated with the activation of CD8+ cytotoxic cells, ${ }^{23,24}$ the presence of lymphadenitis may serve as a positive diagnostic indicator of suspected SCARs in children.

On the other hand, thrombocytopenia and eosinophilia were observed in many cases, though atypical lymphocytosis was not as common as leukocytosis or leucopenia. Recently, 
in addition to SCARs caused by drugs, it has been proposed that infections, such as those resulting from mycoplasma or viruses, might cause SCARs. ${ }^{12,25}$ This suggests that pediatric SCARs are heterogeneous, with various etiologies based on patient background.

An additional source of variability and heterogeneity among pediatric SCARs found in the present study was the latency period between drug initiation and the time at which SCARs developed. We found that DRESS had a longer median time (23.5 days) between administration of the first causative drug dose and the onset of symptoms than did the SJS (4.0 days) or TEN ( 6.5 days) groups. Previous studies have reported similar findings, with an average latency period of 22 days in DRESS cases ${ }^{26}$ and a shorter time period for both TEN and SJS than for DRESS, with symptoms generally emerging within the first 7 days of exposure. ${ }^{27-29}$ This longer latency time in DRESS than in TEN/SJS indicate that early signs and symptoms of DRESS (fever, lymphadenopathy, flu-like symptoms, sore throat or dysphagia, burning pain and pruritus) can easily be overlooked or misdiagnosed. ${ }^{26}$

AS for prognoses, TEN was associated with a much higher ICU transfer rate $(50 \%)$ and a much longer median admission period (30.5 days) than SJS. Patients with skin detachment of more than 30\% carry an increased risk of different systemic complication, it is recommended to transfer subjects with severe SJS/TEN. ${ }^{27,30}$ Furthermore, the TEN mortality rate was $25 \%$ ( 1 of 4 cases) compared to a total mortality rate of $2.1 \%$ among the 3 groups. This result is comparable to the mortality rate of $1 \%--5 \%$ for SJS and $25 \%--30 \%$ for TEN ${ }^{28}$ and it shows the need for an urgent and special care for subjects with TEN.

Treatment for pediatric SCARs are primarily based on supportive care- that is, withdrawal of the culprit drug, close monitoring and supportive care. ${ }^{28}$ Other immune-modulating treatments, such as systemic corticosteroids, IVIG and cyclosporines, are commonly used in persistent cases. ${ }^{1}$ In this study, systemic corticosteroid treatment was most commonly adopted, followed by IVIG. Both systemic corticosteroids and IVIG were administered to $27.7 \%$ of the patients assessed. However, in spite of more recent data that finds a favorable outcome from cyclosporine on SCAR progression ${ }^{31}$ and mortality rates, ${ }^{23,32}$ cyclosporine was introduced in only 1 case, which keeps parallel to the fact that no SJS/TEN cases adopted the cyclosporine treatment in the large pediatric study. ${ }^{10}$ Considering that conflicting results and the possible risk associated with systemic corticosteroids in SCARs cases, ${ }^{33-35}$ further studies are warranted to verify the safety and the efficacy of cyclosporine as the primary option for SCAR treatment.

The present study has a few limitations. All patients in the present study were diagnosed with SCARs based on a clinical assessment and most did not undergo a skin biopsy and/ or patch testing for suspected drug sensitivity. Given that we analyzed cases diagnosed at referral tertiary university hospitals, milder cases treated at regional centers, where spontaneous resolution was achieved by a simple discontinuation of the offending drug, were not included. Nevertheless, this study is valuable because it is the first to assess the Korean representative SCARs registry for pediatrics cases, as per the internationally-accepted RegiSCAR criteria.

In conclusion, a high proportion of Korean pediatric SCARs cases occurred in responses to antibiotic and antiepileptic drugs that are commonly or chronically administered orally. The latency period between drug administration and the development of SCARs varied, and nonspecific symptoms, which were indistinguishable from viral or other infections, often 
indicated the early stages of SCAR development. None of the early signs noted could predict SCARs development; additionally, the extent of resulting skin damage could not be used to predict the development of sequelae. Thus, clinical suspicion should remain the primary method of rapidly diagnosing SCARs and providing prompt treatment.

\section{ACKNOWLEDGMENTS}

We specially give thanks to all members of the Korean SCAR registry for their efforts to build up this database.

\section{REFERENCES}

1. Noguera-Morel L, Hernández-Martín Á, Torrelo A. Cutaneous drug reactions in the pediatric population. Pediatr Clin North Am 2014;61:403-26. PUBMED | CROSSREF

2. Gomes ER, Brockow K, Kuyucu S, Saretta F, Mori F, Blanca-Lopez N, et al. Drug hypersensitivity in children: report from the pediatric task force of the EAACI Drug Allergy Interest Group. Allergy 2016;71:149-61. PUBMED | CROSSREF

3. Teo YX, Walsh SA. Severe adverse drug reactions. Clin Med (Lond) 2016;16:79-83. PUBMED | CROSSREF

4. Hoetzenecker W, Nägeli M, Mehra ET, Jensen AN, Saulite I, Schmid-Grendelmeier P, et al. Adverse cutaneous drug eruptions: current understanding. Semin Immunopathol 2016;38:75-86. PUBMED | CROSSREF

5. Chantaphakul H, Sanon T, Klaewsongkram J. Clinical characteristics and treatment outcome of StevensJohnson syndrome and toxic epidermal necrolysis. Exp Ther Med 2015;10:519-24. PUBMED | CROSSREF

6. Blumenthal KG, Wickner PG, Lau JJ, Zhou L. Stevens-Johnson syndrome and toxic epidermal necrolysis: a cross-sectional analysis of patients in an integrated allergy repository of a large health care system. J Allergy Clin Immunol Pract 2015;3:277-280.e1. PUBMED | CROSSREF

7. Mockenhaupt M. International registry of severe cutaneous adverse reactions (SCAR) to drugs and collection of biological sample, study protocol [Internet]. RegiSCAR study group; 2010 [cited 2018 Nov 5]. Available from: http://www.regiscar.org/pdf/regiscarprotocol100312.pdf.

8. Jung HY, Park S, Shin B, Lee JH, Lee SJ, Lee MK, et al. Prevalence and clinical features of drug reactions with eosinophilia and systemic symptoms syndrome caused by antituberculosis drugs: a retrospective cohort study. Allergy Asthma Immunol Res Forthcoming 2019.

9. World Health Organization; Uppsala Monitoring Centre. The use of the WHO-UMC system for standardized case causality assessment [Internet]. Uppsala: The Uppsala Monitoring Centre; 2005 [cited 2018 Nov 5]. Available from: http://www.who.int/medicines/areas/quality_safety/safety_efficacy/ WHOcausality_assessment.pdf.

10. Dibek Misirlioglu E, Guvenir H, Bahceci S, Haktanir Abul M, Can D, Usta Guc BE, et al. Severe cutaneous adverse drug reactions in pediatric patients: a multicenter study. J Allergy Clin Immunol Pract 2017;5:757-63. PUBMED | CROSSREF

11. Kardaun SH, Sidoroff A, Valeyrie-Allanore L, Halevy S, Davidovici BB, Mockenhaupt M, et al. Variability in the clinical pattern of cutaneous side-effects of drugs with systemic symptoms: does a DRESS syndrome really exist? Br J Dermatol 2007;156:609-11. PUBMED | CROSSREF

12. Shiohara T, Iijima M, Ikezawa Z, Hashimoto K. The diagnosis of a DRESS syndrome has been sufficiently established on the basis of typical clinical features and viral reactivations. Br J Dermatol 2007;156:1083-4. PUBMED | CROSSREF

13. Park HW, Kim SH, Chang YS, Kim SH, Jee YK, Lee AY, et al. The fas signaling pathway is a common genetic risk factor for severe cutaneous drug adverse reactions across diverse drugs. Allergy Asthma Immunol Res 2018;10:555-61.

PUBMED | CROSSREF 
14. Duong TA, Valeyrie-Allanore L, Wolkenstein P, Chosidow O. Severe cutaneous adverse reactions to drugs. Lancet 2017;390:1996-2011. PUBMED | CROSSREF

15. Fakoya AO, Omenyi P, Anthony P, Anthony F, Etti P, Otohinoyi DA, et al. Stevens - Johnson syndrome and toxic epidermal necrolysis; extensive review of reports of drug-induced etiologies, and possible therapeutic modalities. Open Access Maced J Med Sci 2018;6:730-8. PUBMED | CROSSREF

16. Vernacchio L, Kelly JP, Kaufman DW, Mitchell AA. Medication use among children $<12$ years of age in the United States: results from the Slone Survey. Pediatrics 2009;124:446-54. PUBMED | CROSSREF

17. Wolf R, Orion E, Marcos B, Matz H. Life-threatening acute adverse cutaneous drug reactions. Clin Dermatol 2005;23:171-81.

PUBMED | CROSSREF

18. Demoly P, Adkinson NF, Brockow K, Castells M, Chiriac AM, Greenberger PA, et al. International Consensus on drug allergy. Allergy 2014;69:420-37. PUBMED | CROSSREF

19. Baeck M, Marot L, Nicolas JF, Pilette C, Tennstedt D, Goossens A. Allergic hypersensitivity to topical and systemic corticosteroids: a review. Allergy 2009;64:978-94. PUBMED | CROSSREF

20. Freymond N, Catelain A, Queille E, Augey F, Nicolas JF. Allergic reaction to methylprednisolone. Rev Med Interne 2003;24:698-700 PUBMED | CROSSREF

21. Mockenhaupt M, Viboud C, Dunant A, Naldi L, Halevy S, Bouwes Bavinck JN, et al. Stevens-Johnson syndrome and toxic epidermal necrolysis: assessment of medication risks with emphasis on recently marketed drugs. The EuroSCAR-study. J Invest Dermatol 2008;128:35-44. PUBMED | CROSSREF

22. Wolf R, Matz H, Marcos B, Orion E. Drug rash with eosinophilia and systemic symptoms vs toxic epidermal necrolysis: the dilemma of classification. Clin Dermatol 2005;23:311-4. PUBMED | CROSSREF

23. Khalili B, Bahna SL. Pathogenesis and recent therapeutic trends in Stevens-Johnson syndrome and toxic epidermal necrolysis. Ann Allergy Asthma Immunol 2006;97:272-80. PUBMED | CROSSREF

24. Downey A, Jackson C, Harun N, Cooper A. Toxic epidermal necrolysis: review of pathogenesis and management. J Am Acad Dermatol 2012;66:995-1003. PUBMED | CROSSREF

25. Shiohara T, Kano Y. A complex interaction between drug allergy and viral infection. Clin Rev Allergy Immunol 2007;33:124-33. PUBMED | CROSSREF

26. Kardaun SH, Sekula P, Valeyrie-Allanore L, Liss Y, Chu CY, Creamer D, et al. Drug reaction with eosinophilia and systemic symptoms (DRESS): an original multisystem adverse drug reaction. Results from the prospective RegiSCAR study. Br J Dermatol 2013;169:1071-80. PUBMED | CROSSREF

27. Belver MT, Michavila A, Bobolea I, Feito M, Bellón T, Quirce S. Severe delayed skin reactions related to drugs in the paediatric age group: a review of the subject by way of three cases (Stevens-Johnson syndrome, toxic epidermal necrolysis and DRESS). Allergol Immunopathol (Madr) 2016;44:83-95. PUBMED | CROSSREF

28. Harr T, French LE. Stevens-Johnson syndrome and toxic epidermal necrolysis. Chem Immunol Allergy 2012;97:149-66. PUBMED | CROSSREF

29. Peter JG, Lehloenya R, Dlamini S, Risma K, White KD, Konvinse KC, et al. Severe delayed cutaneous and systemic reactions to drugs: a global perspective on the science and art of current practice. J Allergy Clin Immunol Pract 2017;5:547-63. PUBMED | CROSSREF

30. Mockenhaupt M. The current understanding of Stevens-Johnson syndrome and toxic epidermal necrolysis. Expert Rev Clin Immunol 2011;7:803-13. PUBMED | CROSSREF

31. St John J, Ratushny V, Liu KJ, Bach DQ, Badri O, Gracey LE, et al. Successful use of cyclosporin A for Stevens-Johnson syndrome and toxic epidermal necrolysis in three children. Pediatr Dermatol 2017;34:540-6.

PUBMED | CROSSREF 
32. Kirchhof MG, Miliszewski MA, Sikora S, Papp A, Dutz JP. Retrospective review of Stevens-Johnson syndrome/toxic epidermal necrolysis treatment comparing intravenous immunoglobulin with cyclosporine. J Am Acad Dermatol 2014;71:941-7. PUBMED | CROSSREF

33. Zhu QY, Ma L, Luo XQ, Huang HY. Toxic epidermal necrolysis: performance of SCORTEN and the scorebased comparison of the efficacy of corticosteroid therapy and intravenous immunoglobulin combined therapy in China. J Burn Care Res 2012;33:e295-308. PUBMED | CROSSREF

34. Lee HY, Dunant A, Sekula P, Mockenhaupt M, Wolkenstein P, Valeyrie-Allanore L, et al. The role of prior corticosteroid use on the clinical course of Stevens-Johnson syndrome and toxic epidermal necrolysis: a case-control analysis of patients selected from the multinational EuroSCAR and RegiSCAR studies. Br J Dermatol 2012;167:555-62. PUBMED | CROSSREF

35. Schneck J, Fagot JP, Sekula P, Sassolas B, Roujeau JC, Mockenhaupt M. Effects of treatments on the mortality of Stevens-Johnson syndrome and toxic epidermal necrolysis: a retrospective study on patients included in the prospective EuroSCAR Study. J Am Acad Dermatol 2008;58:33-40.

PUBMED | CROSSREF 